\title{
Considerations on determining the castability of dental casting alloys
}

\author{
Cristian Deac*, Alina Gligor and Lucian Tarnu \\ Lucian Blaga University of Sibiu, Romania, Department of Industrial Engineering and Management, \\ Emil Cioran str., 4, Sibiu, Romania
}

\begin{abstract}
Castability is, along with biocompatibility, one of the most important characteristics of metallic materials used for dental prosthetic applications. In addition, the characteristics and performance of the employed casting machine are also decisive for the end result of the casting, especially when dealing with titanium or a titanium alloy. Starting from a critical analysis of the existing methods for determining the castability of dental alloys, the current paper presents a new method (and associated pattern) for determining the castability. Also, given the castability's dependence on the type of casting machine, the paper includes an analysis of suitable casting machines and suggests some possible improvements.
\end{abstract}

\section{Introduction}

In the last decades, many companies have started producing metal alloys designed specifically for the manufacturing of dental prostheses by casting. However, these dental casting alloys vary greatly in terms of their suitability, especially with regard to their castability and biocompatibility.

A material's castability directly influences the quality of the cast part and can refer to both the completeness of the casting, in the sense of the proportion of the cast part that can be correctly created during the casting process and to the casting precision, i.e. to the minimal dimensions of the details that can be obtained.

The casting of small size parts such as prosthetic crowns and bridges involves a high degree of precision - parts often have wall thicknesses in the range of tenths of millimeters, with tolerances in the range of $\pm 0.05 \ldots \pm 0.1 \mathrm{~mm}$ [1] so a predictable behavior of the alloy employed, in terms of hardness, corrosion resistance and mold filling is an important condition. Any quality issue in a dental prosthetic part can become a major problem and lead to its rejection, causing financial losses due to the time lost (as the faulty part itself can be remelted and the material reused).

While the material itself is thus a very important element to be analyzed, several studies carried out in the last decades have shown that the casting machine being used can also have a significant role in determining the quality of the finite parts.

Therefore, in this paper, the authors have analyzed both the currently available methods for assessing the castability of dental casting alloys and also the currently available casting

${ }^{*}$ Corresponding author: cristian.deac@ulbsibiu.ro 
machines, offering solutions for an improvement of both elements and testing these solutions on consecrated dental alloys.

\section{Assessing the castability of dental casting alloys}

When analyzing the extent of the term "castability", there need to be taken into account several rather different categories of casting defects that can occur in cast parts [2]:

- Investment defects, related to the components of the casting system.

- Pores, determined by the existence of gas bubbles, initially dissolved in the molten

- Incomplete castings, related also to the problem of under-dimensioning.

Among all these, both theoretical studies and practical casting experiments indicate that pores are the main problem in small-size cast parts. The area with the highest probability to generate pores is the junction between the casting pattern (model) and the sprue, although pores could be found also in the casting part itself.

Several different methods have been suggested for determining a castability value or index, most being based on the casting of so-called test patterns of particular shapes and sizes.

The best-known test patterns are the mesh pattern (Figure 1a), the saucer (disc) pattern (Figure 1b), the wedge-type pattern (Figure 1c) and the coil pattern (fig 1d) [1, 3].

The casting of such test patterns of a particular material allows the determining of a castability index for that material. For example, in the case of the saucer pattern, each of the areas A-E has a certain score and the castability index takes into account the completeness of each of these areas in a cast part [4]:

$$
\text { Castability index }(C I)=\frac{\text { number of cast segments }}{25} * 100[\%]
$$

Similarly, for the flat mesh pattern,

$$
\text { Castability index }(C I)=\frac{\text { number of cast segments }}{64} * 100[\%]
$$

Kim [5] has determined that castings with individual sprues offer better results in terms of castability than castings with runner bars or with group sprues.

Koike et al. [6] has analyzed the effects of parameters such as rotation speed and force on the castability of an acute angled wedge, concluding that a wedge angle of $30^{\circ}$ offers better results than a wedge angle of $15^{\circ}$.

Some authors have tried to determine castability by means of theoretical calculations, but based on one or the other test pattern shape.

For example, starting from a plane mesh pattern, Hirano [7] has determined the empirical formula (3) for the castability index.

$$
\mathrm{C}_{\mathrm{v}, \mathrm{t}}=\mathrm{a}+\mathrm{bT}_{\mathrm{m}}^{2} \sqrt{\mathrm{T}_{\mathrm{a}}}
$$

Where:

$$
\mathrm{C}_{\mathrm{v}, \mathrm{t}}=\ln \frac{\frac{2}{3}+\sqrt{\mathrm{C}_{\mathrm{v}}}}{\frac{2}{3}+\sqrt{1-\mathrm{C}_{\mathrm{v}}}}
$$

$\mathrm{C}_{\mathrm{v}}=$ fraction of the total number of mesh segments that were cast entirely;

$\mathrm{T}_{\mathrm{m}}=$ mold preheating temperature;

$\mathrm{T}_{\mathrm{a}}=$ alloy overheating temperature;

$\mathrm{a}, \mathrm{b}=$ material-dependent constants. 
Each of these test patterns intends to facilitate the description of a material's behavior during casting. However, for most test patterns, the information provided is relatively limited or too specific. For example, in the case of the saucer pattern, the applicability is limited by the fixed dimensions of the pattern, by the fact that the casting material has to cover only a relatively short distance until crystallization, but also by the subjectivity stemming from the equal importance granted to the various areas on the test pattern.

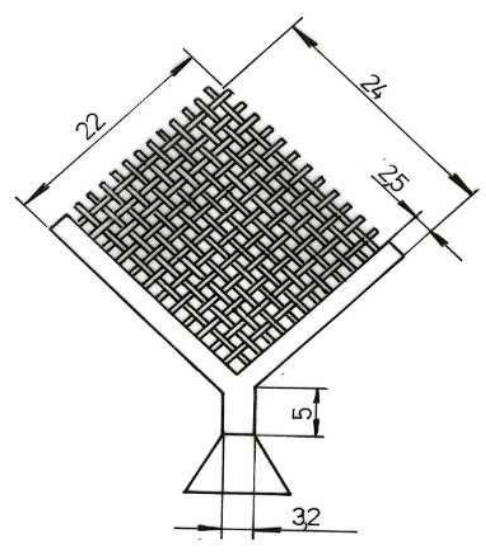

a)

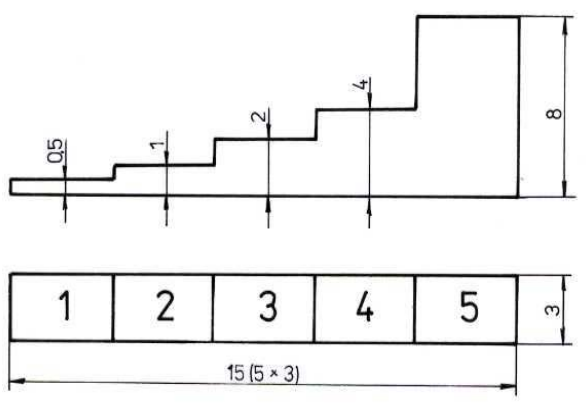

c)
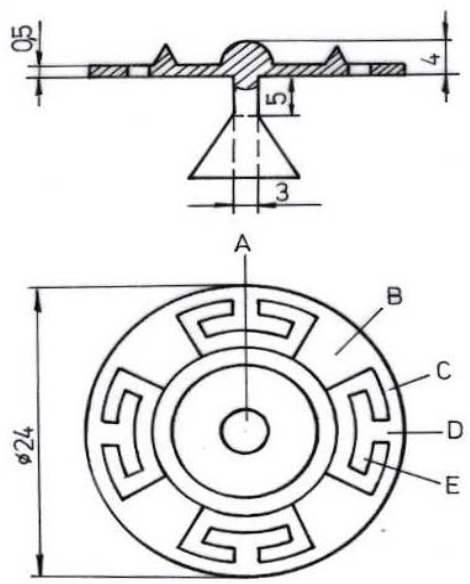

b)

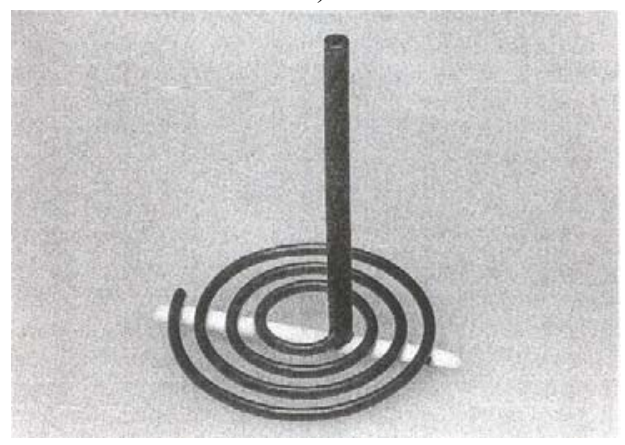

d)

Fig. 1. Some of the test patterns used for the assessment of castability: a) flat mesh pattern; b) saucer pattern; c) wedge pattern d) flat coil pattern.

The D pattern, developed by Deac et al. [1], seeks to eliminate the disadvantages noticed in the other types of casting test patterns. It was conceived to encompass long sinusoidal branches of various diameters that can thus offer information on castability's variation with the material's thickness and with the change in the flowing direction of the molten material.

With the D pattern, the castability of metallic alloys can be assessed by means of the castability coefficient $\mathrm{C}_{\mathrm{t}, \mathrm{di}}$ (for the diameter $\mathrm{d}_{\mathrm{i}}$ of the branch $i$ of the test pattern):

$$
C_{t, d i}=\left(5-d_{i}\right) \cdot 5+\left(20-m_{i}\right)+2 n_{i}
$$

Where $d_{i}$ represents the diameter of branch $i, m_{i}$ represents the sinusoidal pitch, while $n_{i}$ represents the number of cast sinusoidal segments (or fragments of segments) resulted on the cast pattern. 


\section{Influence of the casting equipment}

The most widely used technologies for the casting of small parts for dental prosthetic applications are currently the vacuum-pressure method and the centrifugation method.

Several studies have analyzed the influence of the casting method on the castability. For example, Takahashi et al. [8] studying only the castability of titanium, obtained the best results with a centrifugal titanium casting machine, followed by a one-chamber pressure casting machine and then by a two-chamber pressure casting machine.

A comparative analysis of the commercially available casting systems [1] has revealed several disadvantages and limits that none of them can fully eliminate, related on the one hand to the complexity and the costs of the equipment or of the auxiliary materials needed and on the other hand to functional problems such as difficulties in precisely controlling the melting process (especially for the systems using electric arc melting), a relative slowness of the melting process and a limited maximal heating temperature (especially in systems with resistive heating) or difficulties in determining the exact moment for the passage of the molten material into the casting mold and the usage of special melting crucibles (at systems with inductive heating and vacuum-pressure casting).

Based on this, Deac et al. [1] have developed a new casting equipment and procedure, combining inductive melting and inert gas pressure casting under continuous vacuuming of the mold.

The new casting equipment (figure 2) consists of an airtight melting chamber A connected to a vacuuming system and heated by means of a spiral inductor that is placed coaxially around the melting chamber and another airtight chamber B, placed above chamber $\mathrm{A}$ and containing the casting mold 5 .

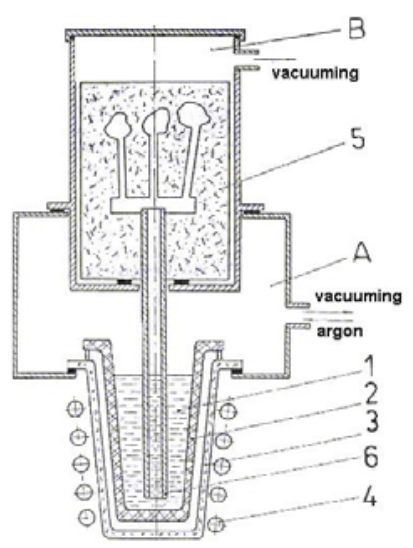

Fig. 2. The new melting and casting equipment: A - melting chamber: 1 - molten metallic material; 2 - melting crucible; 3 - protection part; 4 - inductor; B - casting chamber: 5 - mold; 6 - ceramic connection tube.

\section{Experimental results}

In order to assess the efficiency of the D pattern, the authors have carried out several test castings of D patterns with branches of 1,2 and $2.5 \mathrm{~mm}$, respectively and made of commercially pure titanium (Ti Grade 2), of a titanium alloy. TiAl6V4 (Ti Grade 5) and of a Ni-Cr-based alloy, Wirolloy, all manufactured by Bego GmbH of Germany.

Titanium Grade 2 is essentially $100 \%$ pure titanium. Titanium Grade 5 is a titanium alloy with $90 \%$ titanium, $6 \%$ aluminum and $4 \%$ vanadium, while Wirolloy contains $63.2 \% \mathrm{Ni}$, $23.0 \% \mathrm{Cr}, 9.0 \% \mathrm{Fe}, 3.0 \% \mathrm{Mo}, 1.8 \% \mathrm{Si}$ and less than $0.1 \% \mathrm{C}$. 
The casting equipment used for the experimental researches was based on a casting machine Fornax 35 EM manufactured by Bego GmbH of Germany, that was modified in such a manner as to conform as much as possible to the ideas presented at the end of paragraph 2 and figure 2. Figure 3 shows the interior of the modified casting machine. In order to allow the creation of proper working atmospheres, the machine was connected to a vacuum pump ROV5 and to a pressured argon container.

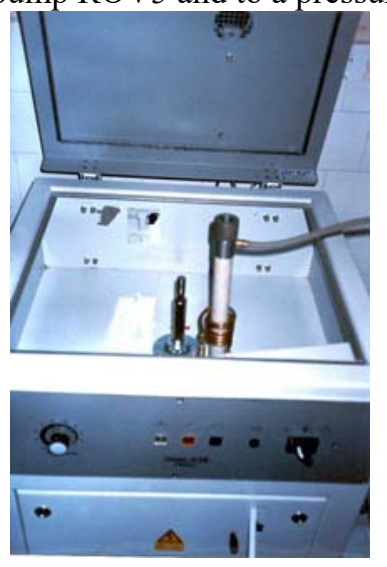

Fig. 3. Interior of the casting equipment used for the experimental researches.

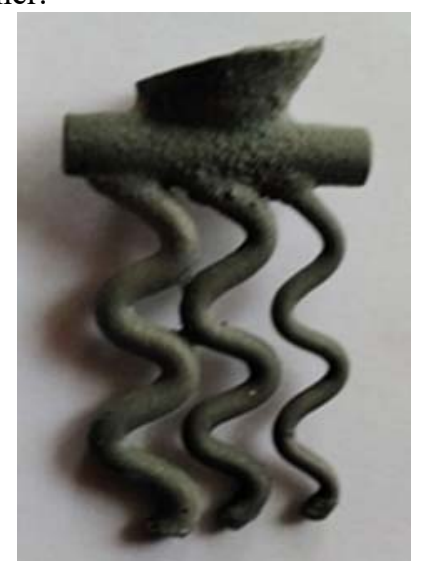

Fig. 4. D pattern cast of Wirolloy.

The castability indices for the three branches of the D pattern cast of Titanium Grade 2, the results were as follows:

$$
\begin{aligned}
& \mathrm{C}_{\mathrm{T} 22.5}=(5-2.5) \cdot 5+(20-18)+2 \cdot 4=22.5 \\
& \mathrm{C}_{\mathrm{T} 22}=(5-2) \cdot 5+(20-18.5)+2 \cdot 4=24.5 \\
& \mathrm{C}_{\mathrm{T} 21.5}=(5-1.5) \cdot 5+(20-19)+2 \cdot 4=26.5
\end{aligned}
$$

while for the pattern made of Titanium Grade 5 the results were as follows:

$\mathrm{C}_{\mathrm{T} 52.5}=(5-2.5) \cdot 5+(20-18.5)+2 \cdot 4=22$

$\mathrm{C}_{\mathrm{T} 52}=(5-2) \cdot 5+(20-19)+2 \cdot 4=24$

$\mathrm{C}_{\mathrm{T} 51.5}=(5-1.5) \cdot 5+(20-19)+2 \cdot 4=26.5$

and finally, the pattern cast of Wirolloy offered the best results:

$\mathrm{C}_{\mathrm{Wi2} .5}=(5-2.5) \cdot 5+(20-18)+2 \cdot 5=24.5$

$\mathrm{C}_{\mathrm{Wi2}}=(5-2) \cdot 5+(20-18)+2 \cdot 5=27$

$\mathrm{C}_{\text {Wi1. }}=(5-1.5) \cdot 5+(20-18)+2 \cdot 5=29.5$,

The comparative results for the castability of the three materials are given in the graph in figure 5 .

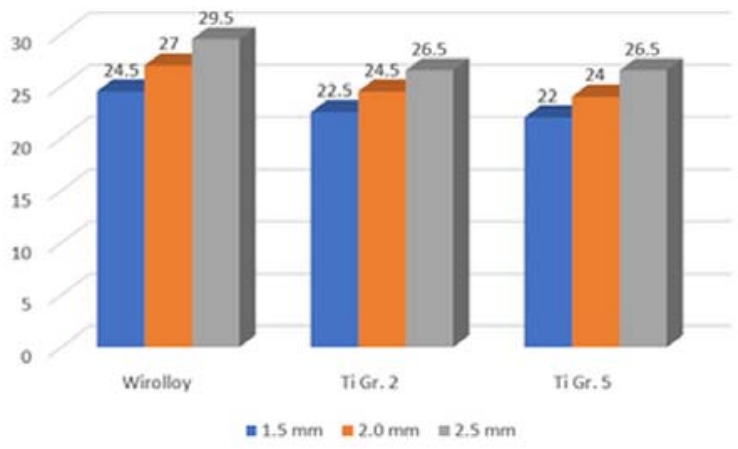

Fig. 5. Comparative results for the castability of the three materials determined using $D$ patterns and the modified Fornax 35 EM casting machine. 


\section{Conclusions}

As has been shown, the castability of dental alloys can be determined by means of test patterns. While many of these have various disadvantages, the $\mathrm{D}$ pattern eliminates most of these drawbacks and can be used successfully to test the castability of dental alloys.

The experimental tests carried out by casting D patterns on a specially modified casting machine have proven that "classical" dental alloys such as Wirolloy have a better castability than titanium or the titanium alloy Ti6Al4V, a fact consistent with results reported by other authors $[3,7,8]$. This can be attributed to the difficulties brought by the melting and casting of titanium, a metal that has a high chemical reactivity, especially towards oxygen and nitrogen.

In future, the authors intend to conduct further researches on the one hand in order to improve the methodology of determining the castability using a D-like pattern and on the other hand in order to optimize the components of the casting system and the employed casting technology, so that better results, in terms of castability, can be achieved even for difficult dental alloys such as titanium and titanium alloys.

\section{References}

1. C. Deac, Contributions to the technological development of the melting and casting of metallic materials used in dental technics (Translated from Romanian) ("Lucian Blaga" University of Sibiu, Sibiu, 2003)

2. R. Marxkors, Deut. Zahnaerztl. Z., 54, 600 (1999)

3. I. Watanabe, M. Woldu, K. Watanabe, T. Okabe, J. Mater. Sci. - Mater. M., 101, 547 (2000)

4. J. M. Meyer, H. Lüthy, Quint. Zahnt., 21, 681 (1995)

5. S.T. Kim, M.S. Vang, H.S. Yang, S.W. Park, H.O. Park, H.P. Lim, J. Korean Acad. Prosthodont, 45, 123 (2007)

6. M. Koike, A. Krysiak, K.S. Chan, L. Guo and T. Okabe, J. Mater. Process. Tech., 211, 224 (2011)

7. S. Hirano, Dent. Mater., 3, 307 (1987)

8. J. Takahashi, J.-Z. Zhang, M. Okazaki, Dent. Mater. J., 12, 245 (1993) 